\title{
APPLICATIONS OF ANALYSIS ON NILPOTENT GROUPS TO PARTIAL DIFFERENTIAL EQUATIONS1
}

\author{
BY G. B. FOLLAND
}

The last few years have witnessed the birth of a body of techniques for obtaining refined regularity theorems for certain hypoelliptic differential operators through analysis of homogeneous convolution operators on nilpotent Lie groups. (Recall that a differential operator $L$ is hypoelliptic if whenever $u$ and $f$ are distributions satisfying $L u=f, u$ must be $C^{\infty}$ on any open set where $f$ is $C^{\infty}$.) The purpose of this paper is to present an outline of the development of this theory, beginning with some background and motivation and sketching the main results and the principal highlights of the methods. No proofs will be given, and the theorems will sometimes be stated in less than maximum generality for the sake of brevity.

The principal applications of the theory we are about to discuss occur in the context of two developments of the 1960's: Kohn's work on the $\bar{\partial}$-Neumann problem and the $\bar{\partial}_{b}$ complex, and Hörmander's work on sums of squares of vector fields. We shall therefore give a brief review of these matters, which will serve as motivation and provide us with some terminology to be used later. (The original papers are Kohn [13], [14] and Hörmander [11]; see also Folland and Kohn [7] for a comprehensive exposition of Kohn's work and Kohn [15] for a simplified proof of Hörmander's main theorem.)

We first describe Hörmander's theorem. Let $M$ be a $C^{\infty}$ manifold, and let $X_{0}, X_{1}, \ldots, X_{n}$ be real $C^{\infty}$ vector fields on $M$. By a commutator of order $k$ of the $X_{j}$ 's we shall mean a vector field of the form

$$
\left[X_{i_{1}},\left[X_{i_{2}}, \ldots\left[X_{i_{k-1}}, X_{i_{k}}\right] \ldots\right]\right], \quad 0 \leqslant i_{j} \leqslant n .
$$

We shall say that $X_{0}, \ldots, X_{n}$ satisfy the Hörmander condition of order $m$ if the $X_{j}$ 's and their commutators of order $\leqslant m$ span the tangent space to $M$ at every point.

THEOREM (HöRMANDER). If $X_{0}, \ldots, X_{n}$ satisfy the Hörmander condition of order $m$ for some $m \geqslant 1$, then the operator

is hypoelliptic.

$$
L=\sum_{1}^{n} X_{j}^{2}+X_{0}
$$

Invited address presented at the 741 st meeting of the Society at Albuquerque, November 20 , 1976; received by the editors December 13, 1976.

AMS (MOS) subject classifications (1970). Primary 35-02, 35H05, 43A80; Secondary 35B45, $35 \mathrm{~N} 15,44 \mathrm{~A} 25$.

1 Partially supported by NSF Grant MCS76-06325. 
(Note that, in particular, one can take $X_{0}=0$, which yields the pure sumof-squares situation.)

Now we turn to Kohn's work. Let $V$ be a Hermitian complex manifold of complex dimension $n \geqslant 2$, and let $M$ be a real $C^{\infty}$ hypersurface in $V$. We assume that $M$ is given as the locus of zeros of a $C^{\infty}$ real-valued function $r$ defined on a neighborhood of $M$ such that $d r \neq 0$ on $M$. (In case $M$ is the boundary of a bounded domain $D \subset V$, we assume that $r<0$ in $D$.) The complex tangent bundle CTM then has two distinguished subbundles denoted by $T_{1,0} M$ and $T_{0,1} M$, namely, the intersections of $C T M$ with the holomorphic and antiholomorphic tangent bundles on $V$. These both have dimension $n-1$, so their sum has codimension one in CTM.

The Levi form of $M$ is the Hermitian form $\langle,\rangle_{L}$ defined on each fiber of $T_{1,0} M$ by

$$
\left\langle\xi_{1}, \xi_{2}\right\rangle_{L}=2\left\langle\partial \bar{\partial} r, \xi_{1} \wedge \bar{\xi}_{2}\right\rangle
$$

The significance of the Levi form in our context arises from the following equivalent definition: if $Z_{1}, Z_{2}$ are smooth sections of $T_{1,0} M$, the function $\left\langle Z_{1}, Z_{2}\right\rangle_{L}$ is given by

$$
\left\langle Z_{1}, Z_{2}\right\rangle_{L}=\left\langle\partial r,\left[Z_{1}, \bar{Z}_{2}\right]\right\rangle .
$$

The one-form $\partial r$ annihilates $T_{1,0} M$, so the nonvanishing of the Levi form means that $T_{1,0} M \oplus T_{0,1} M$ is not closed under Lie brackets. We shall be concerned with the following three conditions on the Levi form:

Condition $Z(q)$. The Levi form has at least $n-q$ positive eigenvalues or at least $q+1$ negative eigenvalues at each point.

Condition $Y(q)$. The Levi form satisfies condition $Z(q)$ and condition $Z(n-1-q)$.

Strong pseudoconvexity. The Levi form is positive definite at each point. (This implies condition $Z(q)$ for $1 \leqslant q \leqslant n$.)

Let $B^{q}$ be the space of distribution sections of the $q$ th exterior power of $\left(T_{0,1} M\right)^{*}$. By throwing away the component of the $\bar{\partial}$ operator which is normal to $M$, one obtains the tangential Cauchy-Riemann operator $\bar{\partial}_{b}: B^{q} \rightarrow B^{q+1}$. Let $\vartheta_{b}: B^{q+1} \rightarrow B^{q}$ be its formal adjoint with respect to the given metric, and let $\square_{b}=\bar{\partial}_{b} \vartheta_{b}+\vartheta_{b} \overline{\partial_{b}}$ be the associated "Laplacian". Unlike the ordinary Laplacian, $\square_{b}$ is not elliptic: its characteristic variety is the orthogonal complement of $T_{1,0} M \oplus T_{0,1} M$ in $(\mathrm{C} T M)^{*}$. However, by equation (1) the nonvanishing of the Levi form allows one to obtain some control over the "weak" direction by considering commutators of derivatives in the "elliptic" directions $T_{1,0} M \oplus$ $T_{0,1} M$.

In fact, let $L_{k}^{2}\left(B^{q}\right.$, loc $)$ be the space of elements of $B^{q}$ which, together with their derivatives of order $\leqslant k$, are in $L^{2}$ on compact subsets of $M$. We then have the following regularity theorem.

THEOREM (KoHN). Suppose $M$ satisfies condition $Y(q)$. Then $\square_{b}$ is hypoelliptic on $B^{q}$; more precisely, if $\square_{b} \phi=\psi$ where $\psi \in L_{k}^{2}\left(B^{q}\right.$, loc $)$, then 


$$
\phi \in L_{k+1}^{2}\left(B^{q}, \text { loc }\right) .
$$

Thus, solving the equation $\square_{b} \phi=\psi$ gains one derivative. This is "half as good" as elliptic regularity, which (for a second order operator) entails the gain of two derivatives.

Next, suppose that $M$ is the boundary of a relatively compact domain $D \subset V$. Let $A^{q}$ be the space of $C^{\infty}(0, q)$-forms on $\bar{D}=D \cup M$, and let $A_{M}^{q}$ be the space of their restrictions to $M$. As above, we consider the operator $\overline{\mathrm{\partial}}: A^{q} \rightarrow A^{q+1}$, its adjoint $\vartheta: A^{q+1} \rightarrow A^{q}$, and the "Laplacian" $\square=\bar{\partial} \boldsymbol{\vartheta}+\boldsymbol{\vartheta} \overline{\mathrm{\partial}}$. Also, if $\phi \in A^{q}$, let $\nu \phi \in A_{M}^{q-1}$ be the contraction of $\phi$ with $\bar{\partial} r$ along $M . \phi$ is said to satisfy the $\bar{\partial}-N e u m a n n$ conditions if $\nu \phi=\nu \bar{\partial} \phi=0$. Given $\psi \in A^{q}$, the $\bar{\partial}-$ Neumann problem for $\psi$ is to solve $\square \phi=\psi$ where $\phi$ satisfies the $\bar{\partial}$-Neumann conditions. (We are being a bit imprecise here. We with to consider $\psi$ 's which are merely square-integrable; if $\psi$-and hence $\phi$-is not smooth, the boundary conditions are to be satisfied in a suitable weak sense.) Here $\square$ is elliptic, but the $\bar{\partial}$-Neumann conditions are noncoercive. This is related to the nonellipticity of $\square_{b}$ and poses the same difficulties in proving regularity. The result is the same: one obtains a regularity theorem that is "half as good" as coercive regularity. Specifically, let $H^{q}$ be the space of all $\phi \in A^{q}$ satisfying $\square \phi=0$ and the $\bar{\partial}$-Neumann conditions, and let $L_{k}^{2}(q)$ be the space of $L^{2}(0, q)$-forms on $D$ whose distribution derivatives of order $\leqslant k$ are also $L^{2}$. We then have

Theorem (KoHN). Suppose $M$ satisfies condition $Z(q)$. Then $H^{q}$ is finite dimensional, and the $\bar{\partial}$-Neumann problem for $\psi \in L_{0}^{2}(q)$ is solvable if and only if $\psi$ is orthogonal to $H^{q}$. In this case, if $\psi \in L_{k}^{2}(q)$ then any solution $\phi$ is in $L_{k+1}^{2}(q)$, and if $\psi \in A^{q}$ then $\phi \in A^{q}$.

In both Hörmander's theorem and Kohn's theorem on $\square_{b}$, one is dealing with a second order operator which gives immediate control of derivatives in certain directions (the span of $X_{0}, \ldots, X_{n}$ in the first case, $T_{1,0} M \oplus T_{0,1} M$ in the second), and one obtains control of the remaining directions by considering commutators of derivatives in the "good" directions. In the $\bar{\partial}$-Neumann problem the same considerations enter, in a more subtle way, in the boundary conditions. It is for this reason that invariant operators on certain non-Abelian Lie groups turn out to be a useful class of models for problems such as these.

The main tool for analyzing differential operators on nilpotent groups is the theory of homogeneous convolution operators. This is rooted in a large body of classical results dealing with the Abelian case, some essential points of which we shall now review. A good general reference for this material is Stein [23].

The heart of the matter is the theory of singular integrals on $\mathbf{R}^{n}$, whose history goes back the better part of a century and which has reached its present form through the efforts of a number of people, including Giraud, Mihlin, and especially Calderón and Zygmund. For our purposes, a singular integral kernel will be a $C^{\infty}$ function $k$ on $\mathbf{R}^{n}-\{0\}$ with the following properties: 
(a) $k$ is homogeneous of degree $-n$, that is, $k(r x)=r^{-n} k(x)$ for all $r>0$ and $x \in \mathbf{R}^{n}-\{0\}$; $=0$.

(b) for some (and hence all) $a, b$ with $0<a<b<\infty, \int_{a<|x|<b} k(x) d x$

If $k$ is a singular integral kernel, by (a) $k$ is not locally integrable at the origin; nonetheless, by virtue of (b) $k$ defines a distribution by taking "principal value" integrals. Precisely, if $\phi \in C_{0}^{\infty}\left(\mathbf{R}^{n}\right)$, the action of the distribution $k$ on $\phi$ is given by

$$
\langle k, \phi\rangle=P V \int k(x) \phi(x) d x=\lim _{\varepsilon \rightarrow 0} \int_{|x|>\varepsilon} k(x) \phi(x) d x .
$$

This makes sense because if $\phi$ is supported in the ball $|x|<R$,

$$
\begin{aligned}
& \lim _{\varepsilon \rightarrow 0} \int_{|x|>\varepsilon} k(x) \phi(x) d x \\
& \quad=\lim _{\varepsilon \rightarrow 0}\left[\int_{\varepsilon<|x|<R} k(x) \phi(x) d x-\phi(0) \int_{\varepsilon<|x|<R} k(x) d x\right] \\
& \quad=\int_{|x|<R}[\phi(x)-\phi(0)] k(x) d x,
\end{aligned}
$$

the last integral being absolutely convergent since $\phi(x)-\phi(0)=O(|x|)$.

Let $\delta$ (here and in the sequel) denote the point mass at the origin. A distribution of the form $K=k+c \delta$ where $k$ is a singular integral kernel and $c \in \mathbf{C}$ will be called a kernel of type zero. One can then consider the convolution operator $T: \phi \rightarrow \phi * K$, which is defined (to begin with) on $C_{0}^{\infty}$ and maps it into $C^{\infty}$. Such operators will be called operators of type zero.

THeORem (CALDERón-ZYGmund). Operators of type zero are bounded on $L^{p}, 1<p<\infty$.

The significance of operators of type zero from our point of view is that they are, in a sense, midway between differential operators and inverses of differential operators. An example may help to clarify this. Consider the inhomogeneous Laplace equation $\Delta u=f$ on $\mathbf{R}^{n}$. If $f \in C_{0}^{\infty}$ (say), we can solve this equation by setting $u=f * N$, where $N$ is the "Newtonian potential",

$$
\begin{aligned}
N(x) & =\frac{\Gamma(n / 2)}{2 \pi^{n / 2}(2-n)}|x|^{2-n} & & (n>2) \\
& =(2 \pi)^{-1} \log |x| & & (n=2) .
\end{aligned}
$$

Let us look at the second derivatives of $u$, that is, the operators

$$
T_{j k}: f \rightarrow \frac{\partial^{2} u}{\partial x_{j} \partial x_{k}}=f * \frac{\partial^{2} N}{\partial x_{j} \partial x_{k}} .
$$

It is not hard to see that the distribution derivative $\partial^{2} N / \partial x_{j} \partial x_{k}$ is a kernel of 
type zero. Hence, by the Calderón-Zygmund theorem, we obtain an $L^{p}$ regularity theorem for the Laplacian: the $L^{p}$ norms of all second derivatives of $u$ can be estimated by the $L^{p}$ norm of $f=\Delta u$. (Proofs of the foregoing assertions about $N$ and its derivatives may be found, for example, in [5].)

The same idea can be used to derive regularity theorems for general elliptic operators. The constant-coefficient case leads to operators of type zero as above. To handle the variable-coefficient case, one needs to consider more general operators of the form

$$
T f(x)=P V \int K(x, x-y) f(y) d y
$$

where $K(x, \cdot)$ is a kernel of type zero for each $x$ and varies smoothly in $x$. (See Calderón-Zygmund [1].) Nowadays, these operators are usually treated in terms of the Fourier transform, in which setting they go under the name of "pseudodifferential operators of order zero". This formulation is very convenient for the $L^{2}$ theory, but it is not so useful for studying other function spaces.

In this setting one also finds kernels of other degrees of homogeneity. If $\lambda>0$, let us define a kernel of type $\lambda$ to be a function $K \in C^{\infty}\left(\mathbf{R}^{n}-\{0\}\right)$ which is homogeneous of degree $\lambda-n$. (For example, the Newtonian potential defined by (2) is a kernel of type 2 when $n>2$.) Convolution with a kernel of type $\lambda$ will be called an operator of type $\lambda$. There are various boundedness theorems for operators of type $\lambda$, about which we shall have more to say later.

Now nilpotent groups can be brought into the picture.

Let $g$ be a real finite-dimensional Lie algebra. A family of dilations on $g$ is a one-parameter group $\left\{\gamma_{r}\right\}_{r>0}$ of algebra automorphisms of $g$ of the form $\gamma_{r}=\exp (A \log r)$ where $A$ is a diagonalizable linear transformation of $g$ with positive eigenvalues. (For example, if $g$ is the Abelian Lie algebra $\mathbf{R}^{n}, A=$ identity gives the usual dilations $\gamma_{r}(x)=r x$.) Since replacing $A$ by $c A(c>0)$ amounts merely to a reparametrization of the dilations, we can, and shall, assume that the smallest eigenvalue of $A$ is 1 .

It is easy to see that if $g$ admits a family of dilations, then $g$ must be nilpotent. (However, not every nilpotent Lie algebra admits dilations.) This being the case, if $G$ is the corresponding simply connected Lie group, it is well known that the exponential map is a diffeomorphism from $g$ to $G$ which takes Lebesgue measure on $\mathrm{g}$ to a bi-invariant Haar measure $d x$ on $G$. Moreover, the dilations $\left\{\gamma_{r}\right\}$ induce a family of automorphisms of $G$, still called dilations and denoted by $\left\{\gamma_{r}\right\}$, by the formula exp $\circ \gamma_{r}=\gamma_{r} \circ \exp$. The number $Q=\operatorname{trace}(A)$ is called the homogeneous dimension of $G$. It is the number such that for all $r>0$,

$$
d\left(\gamma_{r} x\right)=r^{Q} d x
$$

With this structure in hand, the standard notions of homogeneity on Euclidean space generalize in the obvious fashion: a function $\phi$ on $G$ is called homogeneous of degree $\lambda$ if $\phi \circ \gamma_{r}=r^{\lambda} \phi$, and a differential operator $L$ is called homogeneous of degree $\lambda$ if $L\left(\phi \circ \gamma_{r}\right)=r^{\lambda}(L \phi) \circ \gamma_{r}$ for all $\phi \in C^{\infty}(G)$. A homogeneous norm on $G$ is a function $x \rightarrow|x|$ from $G$ to $[0, \infty)$ which is $C^{\infty}$ 
away from the origin and homogeneous of degree one and satisfies (a) $|x|=\left|x^{-1}\right|$ for all $x \in G,(b)|x|=0$ if and only if $x=0$. (Here and in what follows, $0=$ the origin = the group identity.) It is easy to see that homogeneous norms always exist; they play the role of the Euclidean absolute value. We shall always assume that our nilpotent groups with dilations are equipped with a fixed homogeneous norm.

As above, we define a kernel of type $\lambda(\lambda>0)$ to be a function which is $C^{\infty}$ away from the origin and homogeneous of degree $\lambda-Q$, and a singular integral kernel to be a function $k$ which is $C^{\infty}$ away from the origin and homogeneous of degree $-Q$ and satisfies

$$
\int_{a<|x|<b} k(x) d x=0 \quad(0<a<b<\infty) .
$$

Just as before, such $k$ 's define distributions via principal value integrals, and we define a kernel of type zero to be a distribution of the form $k+c \delta$. Convolution on the right with a kernel of type $\lambda(0 \leqslant \lambda<Q)$ will then be called an operator of type $\lambda$. The Calderón-Zygmund theorem remains true in this more general setting:

THEOREM. Operators of type zero are bounded on $L^{p}, 1<p<\infty$.

For $p=2$ this theorem is due to Knapp and Stein [12], who were led to study such operators in a completely different context from the present one, that of representations of semisimple Lie groups. In the Abelian case, the $L^{2}$ boundedness of singular integrals can be obtained rather easily by using the Fourier transform, but this tool is not available in the general situation, and Knapp and Stein were forced to invent an entirely new and more delicate proof. Once this was accomplished, it was realized simultaneously by several people that the Calderón-Zygmund technique could be extended to yield the general $L^{p}$ theorem: see Coifman and Weiss [2] and Korányi and Vági [17].

So much for background: now the story really begins. In his lecture at the 1970 International Congress [24], Stein suggested that singular integrals on nilpotent groups should find applications in nonelliptic differential equations and several complex variables. One piece of evidence for this was some results of Korányi and Vági [17] concerning a multidimensional version of the Hilbert transform on the boundary of a certain Siegel domain in $\mathbf{C}^{n}$. Since the latter space will be crucial in what follows, we pause for a moment to describe it.

As we all know, the unit disc $B^{1}$ in $\mathbf{C}$ is holomorphically equivalent to the upper half plane $D^{1}$ via a linear fractional transformation, and the boundary of $D^{1}$-the real axis-can be naturally identified with a group of holomorphic automorphisms of $D^{1}$, namely the horizontal translations. It was discovered by Pjateckir-Sapiro [20] that an analogue of this holds in $\mathbf{C}^{n}$. Namely, let $B^{n}$ be the unit ball in $\mathbf{C}^{n}$, and let

$$
D^{n}=\left\{\left(\zeta_{1}, \ldots, \zeta_{n}\right) \in \mathbf{C}^{n}: \sum_{1}^{n-1}\left|\zeta_{j}\right|^{2}-\operatorname{Im} \zeta_{n}<0\right\} .
$$


Then the linear fractional transformation $F$ defined by

$$
F\left(\zeta_{1}, \ldots, \zeta_{n}\right)=\left(1+i \zeta_{n}\right)^{-1}\left(\zeta_{1}, \ldots, \zeta_{n-1}, \zeta_{n}+i\right)
$$

maps $B^{n}$ holomorphically onto $D^{n}$. Now consider the nilpotent group $H_{n-1}$, the Heisenberg group of degree $n-1$, whose underlying manifold is $\mathbf{C}^{n-1} \times \mathbf{R}$ with coordinates $\left(z_{1}, \ldots, z_{n-1}, t\right)=(z, t)$ and whose group law is given by

$$
(z, t) \cdot\left(z^{\prime}, t^{\prime}\right)=\left(z+z^{\prime}, t+t^{\prime}+2 \operatorname{Im} \sum z_{j} \bar{z}_{j}^{\prime}\right) .
$$

$H_{n-1}$ acts on $\mathbf{C}^{n}$ by holomorphic affine transformations which preserve $D^{n}$ and its boundary $\partial D^{n}$ by the formula

$$
(z, t) \cdot\left(\zeta_{1}, \ldots, \zeta_{n}\right)=\left(\zeta_{1}+z_{1}, \ldots, \zeta_{n-1}+z_{n-1}, \zeta_{n}+t+i|z|^{2}+2 i \sum_{1}^{n-1} \zeta_{j} \bar{z}_{j}\right) .
$$

This action is simply transitive on $\partial D^{n}$, so $H_{n-1}$ can be identified with $\partial D^{n}$ by the correspondence

$$
(z, t) \leftrightarrow(z, t) \cdot(0, \ldots, 0)=\left(z_{1}, \ldots, z_{n-1}, t+i|z|^{2}\right) .
$$

Since the action of $H_{n-1}$ on $\mathbf{C}^{n}$ is holomorphic, it commutes with the action of $\bar{\partial}_{b}$ on $\partial D^{n}$. If we transfer the $\bar{\partial}_{b}$ complex to $H_{n-1}$ by the above correspondence, therefore, we see that it is left-invariant, and hence so is the Laplacian $\square_{b}$ formed with respect to a left-invariant metric on $H_{n-1}$.

For future reference, we also note the following facts. First, $H_{n-1}$ has a natural family of dilations $\left\{\gamma_{r}\right\}$ given by

$$
\gamma_{r}(z, t)=\left(r z, r^{2} t\right)
$$

Second, the vector fields

$$
Z_{j}=\frac{\partial}{\partial z_{j}}+i \bar{z}_{j} \frac{\partial}{\partial t}, \quad \bar{Z}_{j}=\frac{\partial}{\partial \bar{z}_{j}}-i z_{j} \frac{\partial}{\partial t}, \quad T=\frac{\partial}{\partial t}
$$

form a basis for the complexified Lie algebra of $H_{n-1}$. The $Z_{j}$ 's span $T_{1,0} H_{n-1}$ and the $\bar{Z}_{j}$ 's span $T_{0,1} H_{n-1}$. (Also, if we set $P_{j}=\left(Z_{j}-\bar{Z}_{j}\right) / 4 i$ and $Q_{j}$ $=\left(Z_{j}+\bar{Z}_{j}\right) / 4$, then $P_{1}, \ldots, P_{n-1}, Q_{1}, \ldots, Q_{n-1}$, and $T$ are a basis for the Lie algebra of $H_{n-1}$, whose only nontrivial commutation relations are

$$
\left[P_{j}, Q_{j}\right]=T \quad(j=1, \ldots, n-1) .
$$

These are the "canonical commutation relations" for position and momentum in quantum mechanics: hence the name "Heisenberg group".)

To return to the historical account: in the spring of 1972 two new developments took place. First, Stein [25] investigated some nonisotropic Lipschitz spaces on complex domains and pointed out the similarity to the nonisotropic homogeneity found on nilpotent groups. Second, following a 
suggestion of C. Fefferman, I computed a fundamental solution for the operator

$$
L=-\frac{1}{2} \sum_{1}^{n-1}\left(Z_{j} \bar{Z}_{j}+\bar{Z}_{j} Z_{j}\right)
$$

on $H_{n-1}$ (which is the real part of $\square_{b}$ acting on functions), and this fundamental solution turned out to be a kernel of type 2. (See [3]. Recall that a fundamental solution for the operator $L$ is a distribution $K$ satisfying $L K=\delta$, where $\delta$, as usual, is the point mass at 0 .) At the Williamstown conference that summer, Stein and I compared notes on these matters and began the collaboration which resulted in the paper [8], in which we proved sharp regularity theorems for $\bar{\partial}_{b}$ on strongly pseudoconvex hypersurfaces. (Actually, we treated the more general case of a nondegenerate Levi form.)

Roughly, the ideas in that paper are the following. (The results and techniques will be described more explicitly in a general context further on.) First we considered the boundary of the domain $D^{n}$, identified with the Heisenberg group $H_{n-1}$. The operator $\square_{b}$ on $q$-forms is easily computed there, and it turns out to be the tensor product of a scalar operator (depending on $q$ ) with the identity map on $q$-forms. Extending the ideas of [3], we computed fundamental solutions for these operators in the cases where they existnamely, when $0<q<n-1$, the allowable range in Kohn's theorem. These fundamental solutions are kernels of type 2, and it follows that if one applies to them two derivatives in the span of $Z_{1}, \ldots, Z_{n-1}, \bar{Z}_{1}, \ldots, \bar{Z}_{n-1}$ (which are homogeneous of degree 1 ) or one derivative in the orthogonal direction $T$ (which is homogeneous of degree 2), one obtains kernels of type zero. By means of the $L^{p}$ boundedness theorems for these kernels we obtained regularity theorems for $\square_{b}$ which improved Kohn's result in two respects: they dealt with $L^{p}, 1<p<\infty$, instead of just $L^{2}$, and they gave a gain of two derivatives in the complex directions as well as one derivative overall. We also obtained some results on Lipschitz (or Hölder) continuity.

Now suppose $M$ is an arbitrary strongly pseudoconvex hypersurface in a complex $n$-manifold $V$. Given $x \in M$, there is a biholomorphic map $F_{x}$ from a neighborhood $U$ of $x$ in $V$ to $\mathbf{C}^{n}$ such that $F_{x}(x)=0$ and $F_{x}(U \cap M)$ has third-order contact with the Heisenberg group $H_{n-1} \cong \partial D^{n}$ at 0 . One can choose the maps $F_{x}$ to depend smoothly on $x$, and one can choose a metric on $M$ so that $F_{x}$ is essentially an isometry at $x$. Under these conditions, in the coordinates given by $F_{x}$, the Laplacian $\square_{b}$ on $M$ near $x$ will look very much like $\square_{b}$ on $H_{n-1}$ near 0 , and the fundamental solution for the latter, composed with $F_{x}$, will become an approximate fundamental solution or parametrix for the former.

Stein and I actually used a somewhat different construction, but the above remarks give the motivation. Under suitable restrictions on the metric, we did construct a parametrix for $\square_{b}$ on $M$ by "locally approximating $M$ with a Heisenberg group", and from this we extended the regularity theorems on $H_{n-1}$ to the general case. 
From this work emerged the following general philosophy concerning the theory of differential operators constructed from vector fields whose commutators play an essential role. A natural class of models for noncommuting vector fields is the left-invariant vector fields on non-Abelian Lie groups. To study a set of noncommuting vector fields, then, one should find a group whose Lie algebra mimics the structure of the original vector fields in a suitable sense, and which admits a "harmonic analysis" that will yield results similar to the classical Euclidean case. The nilpotent groups with dilations (or the more special "stratified groups" discussed below) fulfill the second requirement, and they seem to be general enough to fulfill the first in all known cases. One should then perform analysis on the group and somehow transfer the results to the original problem. More specifically, tracing the general outline of [8], one is led to the following three-part program:

I. Show that under appropriate hypotheses, homogeneous left-invariant differential operators on nilpotent groups with dilations have homogeneous fundamental solutions. (Stein and I were lucky to be able to write the solutions down explicitly on the Heisenberg group, but there are good reasons why one should not expect to do this in general. For one thing, the functions involved will of ten not be analytic: cf. [6].)

II. Study homogeneous convolution operators (operators of type $\lambda$ ) on nilpotent groups. In particular, ascertain how they affect various differentiability properties of functions, and combine this with (I) to prove regularity theorems.

III. Construct a theory for approximating more general operators by operators on nilpotent groups and extending the regularity theorems of (II) to the general case.

This program has been fairly extensively carried out by Rothschild, Stein, and myself in [4] and [22]. What follows is a description of the essential features of these papers, according to the above outline.

I. Fundamental solutions. The main result is the following:

THEOREM 1. Let $G$ be a nilpotent group with dilations, of homogeneous dimension $Q$.

(a) Let $L$ be a differential operator on $G$ which is homogeneous of degree $\lambda, 0<\lambda<Q$, such that $L$ and its transpose are both hypoelliptic. Then there is a unique kernel of type $\lambda$ which is a fundamental solution for $L$.

(b) Suppose that for each $\xi$ in some manifold we are given an operator $L_{\xi}$ satisfying the above hypotheses and depending smoothly on $\xi$. Then the fundamental solution of (a) also depends smoothly on $\xi$.

In applying this theorem to second-order operators, verifying the hypoellipticity is usually a matter of using Hörmander's theorem [11] or the KohnNirenberg regularity theorem [16]. The hypothesis $\lambda<Q$ cannot be omitted, as the example of the Laplacian on $\mathbf{R}^{2}$ shows: cf. formula (2) above.

II. Analysis on nilpotent groups. Let $G$ be a nilpotent group with dilations $\left\{\gamma_{r}\right\}$ 
and Lie algebra $g$. For each $\alpha \in \mathbf{R}$ let $V_{\alpha}$ be the subspace of $g$ consisting of those elements which are homogeneous of degree $\alpha$, that is,

$$
V_{\alpha}=\left\{X \in g: \gamma_{r}(X)=r^{\alpha} X \text { for all } r>0\right\} .
$$

Since the $\gamma_{r}$ 's are algebra automorphisms of $g$, we have $\left[V_{\alpha}, V_{\beta}\right] \subset V_{\alpha+\beta}$ for all $\alpha, \beta$. We say that $G$ is stratified if $V_{1}$ generates $g$ as a Lie algebra. We shall denote by $m$ the order of nilpotence (i.e., the length of the lower central series) of $G$. If $G$ is stratified, then, we have $\mathfrak{g}=\oplus_{1}^{m} V_{k}$.

Let $G$ be stratified, and fix a basis $X_{1}, \ldots, X_{n}$ for $Y_{1}$. If $k$ is a positive integer and $I=\left(i_{1}, \ldots, i_{k}\right)$ is a $k$-tuple of integers with $1 \leqslant i_{j} \leqslant n$, we define $X_{I}=X_{i_{1}} X_{i_{2}} \cdots X_{i_{k}}$, and set $|I|=k$. Since the $X_{j}$ 's generate $\mathfrak{g}$, each leftinvariant differential operator on $G$ is a linear combination of $X_{I}$ 's and is homogeneous of degree $k$ if and only if it lies in the span of the $X_{I}$ 's with $|I|=k$.

On stratified groups one can obtain a satisfying theory of differentiability which extends the classical Euclidean theory. The basic heuristic principle is this: if one replaces "derivative of order $k$ " by "homogeneous derivative of degree $k$ ", true statements on $\mathbf{R}^{n}$ become true statements on general stratified groups.

For example, recall the classical Sobolev space $L_{k}^{p}(1<p<\infty, k=0,1$, $2, \ldots$ ), the set of functions in $L^{p}$ whose (distribution) derivatives of order $\leqslant k$ are in $L^{p}$. On the stratified group $G$ the natural analogue is

$$
S_{k}^{p}=\left\{f \in L^{p}: X_{I} f \in L^{p} \text { for }|I| \leqslant k\right\},
$$

which is a Banach space in the obvious way. (The $L^{p}$ norm is, of course, taken with respect to Haar measure.) On $\mathbf{R}^{n}, L_{k}^{p}$ can also be defined in terms of fractional powers of the Laplacian $\Delta$, and in this formulation the definition extends naturally to yield spaces $L_{\alpha}^{p}$ for every $\alpha \in \mathbf{R}$ (cf. Stein [23]). On stratified groups, the role of the Laplacian is played by the operator $L$ $=\sum_{1}^{n} X_{j}^{2}, X_{1}, \ldots, X_{n}$ being a basis for $V_{1}$ as above, and in terms of its fractional powers one can define spaces $S_{\alpha}^{p}$ for every $\alpha \in \mathbf{R}$. This refinement is important for technical treasons, as it allows the use of interpolation theorems, but here we shall generally content ourselves with taking $\alpha$ to be a positive integer.

Of course $G$ is canonically diffeomorphic to the Euclidean space $\mathfrak{g}$, so we can also consider the spaces $L_{\alpha}^{p}(\alpha \in \mathbf{R})$ on $G$. We shall also use the localized versions $L_{\alpha}^{p}(\mathrm{loc})$ and $S_{k}^{p}(\mathrm{loc})$, the spaces of functions $f$ such that $\phi f \in L_{\alpha}^{p}$ (resp. $\phi f \in S_{k}^{p}$ ) for all $\phi \in C_{0}^{\infty}$. As for the relationship between $S_{k}^{p}$ and $L_{\alpha}^{p}$, it is clear that $L_{k}^{p}(\mathrm{loc}) \subset S_{k}^{p}(\mathrm{loc})$, and-since every element of $\mathrm{g}$ is a linear

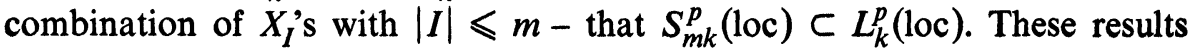
actually extend to arbitrary positive real $k$, and, in particular, we have

THEOREM 2. $L_{k}^{p}(\mathrm{loc}) \subset S_{k}^{p}(\mathrm{loc}) \subset L_{k / m}^{p}(\mathrm{loc})$ for $1<p<\infty, k=1,2,3$, 
The fundamental theorem concerning singular integrals on stratified groups is the following:

THEOREM 3. Operators of type zero are bounded on $S_{k}^{p}(1<p<\infty, k$ $=0,1,2, \ldots)$.

A word about the proof. In the Abelian situation this result follows immediately from the Calderón-Zygmund theorem (the case $k=0$ ), since differentiation commutes with convolution. On non-Abelian groups leftinvariant differentiation does not commute with right convolution, so one needs some extra work in this theorem as well as in other similar situations. The essential ingredient is always some variant of the following:

Lemma. Let $G$ be a stratified group, $X_{1}, \ldots, X_{n}$ a basis for $V_{1}$, and $T$ an operator of type zero. Then there exist operators $T_{j k}$ of type zero $(1 \leqslant j, k \leqslant n)$ such that for all $\phi \in C_{0}^{\infty}$,

$$
X_{j}(T \phi)=\sum_{k=1}^{n} T_{j k}\left(X_{k} \phi\right)
$$

Most of the other regularity theorems for integral operators are essentially local in character. We shall say that an operator $T$ on functions on $G$ is locally bounded from $S_{k}^{p}$ to $S_{k+\lambda}^{p}$ if for every $\psi, \phi_{1}, \phi_{2} \in C_{0}^{\infty}$ with $\phi_{2}=1$ on the support of $\phi_{1}$ there exists a constant $C>0$ such that whenever $f \in S_{k}^{p}$ (loc), we have $T\left(\phi_{1} f\right) \in S_{k+\lambda}^{p}(\mathrm{loc})$ and

$$
\left\|\psi T\left(\phi_{1} f\right)\right\|_{S_{k+\lambda}^{p}} \leqslant C\left\|\phi_{2} f\right\|_{S_{k}^{p}}
$$

The concept of local boundedness on other function spaces is to be interpreted similarly. The following is then an easy consequence of Theorem 3:

THEOREM 4. Operators of type $\lambda(\lambda=1,2, \ldots)$ are locally bounded from $S_{k}^{p}$ to $S_{k+\lambda}^{p}(1<p<\infty, k=0,1,2, \ldots)$.

There is also a corresponding result relative to the classical Sobolev spaces:

THEOREM 5. Operators of type $\lambda(\lambda=1,2,3, \ldots)$ are locally bounded from $L_{\alpha}^{p}$ to $L_{\alpha+(\lambda / m)}^{p}(1<p<\infty, \alpha \geqslant 0)$.

Another important class of function spaces in classical analysis is the Lipschitz spaces $\Lambda_{\alpha}, \alpha>0$ (cf. Stein [23]). The natural analogues of these spaces on stratified groups are the spaces $\Gamma_{\alpha}$ defined as follows. Let $B C$ be the space of bounded continuous functions on the stratified group $G$. If $0<\alpha$ $<1$,

$$
\Gamma_{\alpha}=\left\{f \in B C: \sup _{x, y}|f(x y)-f(x)| /|y|^{\alpha}<\infty\right\},
$$

where $|y|$ is the homogeneous norm of $y$. Also, 


$$
\Gamma_{1}=\left\{f \in B C: \sup _{x, y}\left|f(x y)+f\left(x y^{-1}\right)-2 f(x)\right| /|y|<\infty\right\},
$$

and if $\alpha=\alpha^{\prime}+k$ where $0<\alpha^{\prime} \leqslant 1$ and $k=1,2,3, \ldots$,

$$
\Gamma_{\alpha}=\left\{f \in \Gamma_{\alpha^{\prime}}: X_{I} f \in \Gamma_{\alpha^{\prime}} \text { whenever }|I| \leqslant k\right\} .
$$

As with the Sobolev spaces, we shall consider both $\Gamma_{\alpha}$ and $\Lambda_{\alpha}$ and their localized versions on $G$, and we have the following analogues of Theorems 2 through 5 for them:

THEOREM 6. $\Lambda_{\alpha}(\mathrm{loc}) \subset \Gamma_{\alpha}(\mathrm{loc}) \subset \Lambda_{\alpha / m}(\mathrm{loc})$ for $\alpha>0$.

THEOREM 7. Operators of type zero are locally bounded on $\Gamma_{\alpha}(\alpha>0)$.

(In the Abelian case this is a folk theorem whose idea goes back to Hölder; a simple proof can be found in Calderón and Zygmund [1]. The non-Abelian case, for $0<\alpha<1$, is due to Korányi and Vági [17].)

THEOREM 8. Operators of type $\lambda(\lambda=1,2,3, \ldots)$ are locally bounded from $\Gamma_{\alpha}$ to $\Gamma_{\alpha+\lambda}$ and from $\Lambda_{\alpha}$ to $\Lambda_{\alpha+(\lambda / m)}(\alpha>0)$.

Finally, we have the following generalization of the Sobolev imbedding theorem relating the various spaces $S_{k}^{p}$ and $\Gamma_{\alpha}$ :

THEOREM 9. $S_{k}^{p} \subset S_{j}^{q}$ whenever $1<p<q<\infty$ and $j=k-Q\left(p^{-1}\right.$ $\left.-q^{-1}\right) \geqslant 0$. Also, $S_{k}^{p} \subset \Gamma_{\alpha}$ whenever $\alpha=k-(Q / p)>0$.

Now suppose $L$ is a left-invariant differential operator on the stratified group $G$ satisfying the hypotheses of Theorem 1. Given a function $f$ on $G$, one can solve the equation $L u=f$ on a bounded open set $\Omega$ by taking $u$ to be the convolution of $\phi f$ with the fundamental solution, where $\phi \in C_{0}^{\infty}$ is identically one on $\Omega$. Since the mapping $\phi f \rightarrow u$ is an operator of type $\lambda$ (where $\lambda$ is necessarily an integer), and since any other solution differs from $u$ by a $C^{\infty}$ function on $\Omega$, one obtains local regularity theorems for $L$ in terms of various function spaces by applying Theorems 4,5 , and 8 .

III. Approximating general operators by operators on groups. We shall explain this theory in the context of operators $L=\sum_{1}^{n} X_{j}^{2}$ where the $X_{j}^{\prime}$ s are real vector fields satisfying a Hörmander condition. In [22], Rothschild and Stein do things somewhat more generally in order to handle the $\bar{\partial}_{b}$ complex and operators of Hörmander type with a first order term.

The basic ideas were developed in [8]. However, the general situation presents difficulties which were not encountered there. To see what these are, let us consider the following simple example. Suppose we wish to study the operator $L=X_{1}^{2}+X_{2}^{2}$ on $\mathbf{R}^{2}$, where

$$
X_{1}=\partial / \partial x, \quad X_{2}=x(\partial / \partial y)
$$

Away from the line $x=0, L$ is elliptic, so the natural group associated to the problem is just $\mathbf{R}^{2}$ and the process of approximation by invariant operators is 
just the classical one of freezing the coefficients at a point. However, along the line $x=0$, one needs to use the commutator

$$
Y=\partial / \partial y=\left[X_{1}, X_{2}\right]
$$

in order to span the tangent space. Now $X_{1}, X_{2}$, and $Y$ span a nilpotent Lie algebra which is stratified if we define dilations by

$$
\gamma_{r}\left(X_{1}\right)=r X_{1}, \quad \gamma_{r}\left(X_{2}\right)=r X_{2}, \quad \gamma_{r}(Y)=r^{2} Y,
$$

so the natural group to consider here is the one associated to this Lie algebra (namely, the Heisenberg group $H_{1}$ ). But this group is three dimensional, whereas the original operator acts on a two-dimensional space.

In short, the problems to be overcome are that the "natural" group associated to the vector fields in question (a) may vary from point to point and (b) may be of higher dimension than the original manifold. The solution to the first problem is to use a group which is sufficiently large to reflect the essential commutation relations (plus perhaps some extraneous ones) at every point, and the solution to the second is to lift the original operators to a larger manifold of the same dimension as the group.

In the context of our example, the first point means that we forget about the ellipticity of $L$ away from $x=0$ and use the three-dimensional group throughout. The second point is accomplished as follows: consider the vector fields $\tilde{X}_{1}, \tilde{X}_{2}, \tilde{Y}$ on $\mathbf{R}^{3}$ defined by

$$
\tilde{X}_{1}=\partial / \partial x, \quad \tilde{X}_{2}=(\partial / \partial z)+x(\partial / \partial y), \quad \tilde{Y}=\left[\tilde{X}_{1}, \tilde{X}_{2}\right]=\partial / \partial y .
$$

These vector fields span the tangent space to $\mathbf{R}^{3}$ at every point. Moreover, if we let $\pi: \mathbf{R}^{3} \rightarrow \mathbf{R}^{2}$ be the projection onto the $x y$-plane, it is clear that $\tilde{X}_{j}(f \circ \pi)=\left(X_{j} f\right) \circ \pi$ for all $f \in C^{\infty}\left(\mathbf{R}^{2}\right)$. Hence, if we set $L=\tilde{X}_{1}^{2}+\tilde{X}_{2}^{2}$, we also have

$$
\tilde{L}(f \circ \pi)=(L f) \circ \pi \quad\left(f \in C^{\infty}\left(\mathbf{R}^{2}\right)\right) .
$$

The object then is to study $\tilde{L}$ by using the group associated to the Lie algebra spanned by $\tilde{X}_{1}, \tilde{X}_{2}$, and $\tilde{Y}$, and then to use relation (4) to transfer the results to $L$.

With these ideas in mind we can describe the general lifting and approximation process. Let $X_{1}, \ldots, X_{n}$ be real vector fields on a manifold $M$. We say that $X_{1}, \ldots, X_{n}$ are free up to step $m$ at $x \in M$ if the $X_{j}^{\prime}$ 's and their commutators of order $\leqslant m$ at $x$ satisfy no linear relations but those forced by anticommutativity and the Jacobi identity. Moreover, let $g_{n, m}$ be the free nilpotent Lie algebra of step $m$ on $n$ generators, that is, the quotient of the free Lie algebra on $n$ generators by its $(m+1)$ st commutator ideal, and let $G_{n, m}$ be the corresponding group. Thus $X_{1}, \ldots, X_{n}$ are free up to step $m$ at $x$ if and only if the dimension of the space spanned by the $X_{j}^{\prime}$ 's and their commutators of order $\leqslant m$ at $x$ is the same as that of $G_{n, m}$. We shall denote the generators 
of $g_{n, m}$ by $Y_{1}, \ldots ; Y_{n}$. We note that $g_{n, m}$ has a natural family of dilations $\left\{\gamma_{r}\right\}$ specified by setting $\gamma_{r}\left(Y_{j}\right)=r Y_{j}$, and this family of dilations makes $G_{n, m}$ a stratified group.

Now suppose $X_{1}, \ldots, X_{n}$ are vector fields on a manifold $M$ which satisfy the Hörmander condition of order $m$. Let $k=\operatorname{dim}\left(G_{n, m}\right)-\operatorname{dim}(M)$ and $\tilde{M}$ $=M \times \mathbf{R}^{k}$, and let $\pi: \tilde{M} \rightarrow M$ be the projection onto the first factor. The fundamental lifting theorem is then the following:

THEOREM 10. For each $x \in M$ there exist a neighborhood $U$ of $x$ in $M, a$ neighborhood $U^{\prime}$ of 0 in $\mathbf{R}^{k}$, and vector fields $\tilde{X}_{1}, \ldots, \tilde{X}_{n}$ on $\tilde{U}=U \times U^{\prime} \subset \tilde{M}$ such that:

(a) $\tilde{X}_{j}(f \circ \pi)=\left(X_{j} f\right) \circ \pi$ for all $f \in C^{\infty}(U)$ and $j=1, \ldots, n$;

(b) $\tilde{X}_{1}, \ldots, \tilde{X}_{n}$ are free up to step $m$ on $\tilde{U}$.

Remarks. (1) Since $\operatorname{dim}(\tilde{M})=\operatorname{dim}\left(G_{n, m}\right)$, (b) implies that $\tilde{X}_{1}, \ldots, \tilde{X}_{n}$ satisfy the Hörmander condition of order $m$ on $\tilde{U}$. (2) In [22] this theorem is proved by a rather intricate induction procedure; a somewhat more straightforward proof has since been discovered by Goodman [9].

The next step is to approximate the lifted vector fields $\tilde{X}_{1}, \ldots, \tilde{X}_{n}$ by the left-invariant vector fields $Y_{1}, \ldots, Y_{n}$ on $G_{n, m}$. In order to state the result we need some more terminology. Set $Y_{j 1}=Y_{j}$ for $j=1, \ldots, n$, and for $k=2$, $\ldots, m$ let $Y_{1 k}, Y_{2 k}, \ldots$ be a maximal linearly independent subset of the commutators of the $Y_{j}^{\prime}$ s of order $k$. Thus the $Y_{j k}$ 's all together form a basis for $\mathfrak{g}_{n, m}$. Taking coordinates with respect to this basis and composing with the exponential map, we obtain a coordinate system on $G_{n, m}$ in which the coordinate functions are all homogeneous (of degrees $1, \ldots, m$ ).

Now, any vector field $X$ on $G_{n, m}$ can be written as

$$
X=\sum a_{j k} Y_{j k}, \quad a_{j k} \in C^{\infty} .
$$

If we expand the $a_{j k}$ 's in their Taylor series about 0 in the coordinates defined above, $X$ will be exhibited as a formal sum of homogeneous differential operators. (Note that if $f$ is a homogeneous function of degree $i$, then $f Y_{j k}$ is a homogeneous operator of degree $k-i$.) We say that $X$ is of local degree $\leqslant \lambda$ if each term in this formal sum is homogeneous of degree $\leqslant \lambda$.

The approximation theorem for the lifted vector fields $\tilde{X}_{1}, \ldots, \tilde{X}_{n}$ is then the following. We retain the notation of Theorem 10 (actually the neighborhood $\tilde{U}$ may need to be shrunk somewhat).

THeOREM 11. There exist a neighborhood $V$ of 0 in $G_{n, m}$ and a $C^{\infty}$ mapping $\Theta: \tilde{U} \times \tilde{U} \rightarrow V$ with the following properties:

(a) $\Theta(\eta, \xi)=\Theta(\xi, \eta)^{-1}$, in particular, $\Theta(\xi, \xi)=0$.

(b) For each fixed $\xi \in \tilde{U}$, the map $\Theta_{\xi}: \eta \rightarrow \Theta(\xi, \eta)$ is a diffeomorphism from a neighborhood of $\xi$ to a neighborhood of 0 .

(c) For each $\xi \in \tilde{U}$ and $j=1, \ldots, n,\left(\Theta_{\xi}\right)_{*} \tilde{X}_{j}=Y_{j}+R_{j}$, where $R_{j}$ is of local degree $\leqslant 0$. 
Remarks. (1) Here $\left(\Theta_{\xi}\right)_{*}$ is the map of tangent bundles induced by $\Theta_{\xi}$. (2) In the special case $\tilde{U}=G_{n, m}$ and $\tilde{X}_{j}=Y_{j}$, the map $\Theta$ is merely given by $\Theta(\xi, \eta)=\xi^{-1} \eta$, and $R_{j}=0$. (3) Since $Y_{j}$ is homogeneous of degree 1, (c) says that $\left(\Theta_{\xi}\right)_{*} X_{j}$ is a "small perturbation" of $Y_{j}$ near the origin.

The eventual object of all this is to study the operator $L=\Sigma_{1}^{n} X_{j}^{2}$ on $M$. All considerations are local, so we restrict attention to the neighborhood $U$ of Theorem 10. First, we look at the lifted operator $\tilde{L}=\Sigma_{1}^{n} \tilde{X}_{j}^{2}$ on $\tilde{U}$.

In a sense made precise by Theorem 11 , near each point $L$ is a small perturbation of the operator $L_{0}=\sum_{1}^{n} Y_{j}^{2}$ on $G_{n, m}$. Let $K_{0}$ be the fundamental solution for $L_{0}$ given by Theorem 1 . (This depends on the choice of Haar measure. We fix the Haar measure as the one determined by the metric which makes the basis $\left\{Y_{j k}\right\}$ for $\mathrm{g}_{n, m}$ described above orthonormal. At the same time, let $\left\{\tilde{X}_{j k}\right\}$ be the frame for the tangent bundle of $\tilde{U}$ formed from the $\tilde{X}_{j}$ 's and their commutators in the same way as $\left\{Y_{j k}\right\}$ is formed from the $Y_{j}$ s and their commutators. We impose the metric on $\tilde{U}$ which makes $\left\{\tilde{X}_{j k}\right\}$ orthonormal and we denote by $d \eta$ the induced measure on $\tilde{U}$.) Choose $\psi, \psi^{\prime} \in C^{\infty}(\tilde{U})$ with $\psi^{\prime}=1$ on the support of $\psi$, and define the function $\tilde{K}$ on $\tilde{U} \times \tilde{U}$ by

$$
\tilde{K}(\xi, \eta)=\psi(\xi) K_{0}(\Theta(\eta, \xi)) \psi^{\prime}(\eta)
$$

Let $\tilde{T}$ be the integral operator on $\tilde{U}$ with kernel $\tilde{K}$, that is,

$$
\tilde{T} f(\xi)=\int \tilde{K}(\xi, \eta) f(\eta) d \eta \text {. }
$$

Then $\tilde{T}$ turns out to be a parametrix for $\tilde{L}$, in the following sense. Define the operators $\tilde{S}$ and $\tilde{S^{\prime}}$ by

$$
\tilde{S} f=\tilde{L} \tilde{T} f-\psi f, \quad \tilde{S^{\prime}} f=\tilde{T} \tilde{L} f-\psi f .
$$

Then $\tilde{S}$ and $\tilde{S}^{\prime}$ are integral operators on $\tilde{U}$ whose kernels $\tilde{F}$ and $\tilde{F}^{\prime}$ behave along the diagonal like kernels of type 1 . That is,

$$
\tilde{F}(\xi, \eta)=a(\xi) F_{0}(\Theta(\eta, \xi)) a^{\prime}(\eta)+\text { lower order terms, }
$$

where $a, a^{\prime} \in C_{0}^{\infty}(\tilde{U})$ and $F_{0}$ is a kernel of type 1 ; similarly for $\tilde{F}^{\prime}$.

Thus $\tilde{S}$ and $\tilde{S^{\prime}}$ are roughly "operators of type 1 ", whereas $\tilde{T}$ is roughly an "operator of type 2 ", and the boundedness theorems for homogeneous operators on stratified groups can be carried over to operators like these. Specifically, one has the classical function spaces $L_{\alpha}^{p}$ and $\Lambda_{\alpha}$ on $\tilde{U}$, and one defines $S_{k}^{p}$ on $\tilde{U}$ just as on stratified groups, replacing the basis for $V_{1}$ by the vector fields $\tilde{X}_{1}, \ldots, \tilde{X}_{n}$. Then $\tilde{S}$ and $\tilde{S}^{\prime}$ map $L_{\alpha}^{p}, \Lambda_{\alpha}$, and $S_{k}^{p}$ into $L_{\alpha+(1 / m)}^{p}$, $\Lambda_{\alpha+(1 / m)}$, and $S_{k+1}^{p}$, respectively, while $T$ maps them into $L_{\alpha+(2 / m)}^{p}, \Lambda_{\alpha+(2 / m)}$, and $S_{k+2}^{p}$, respectively. (One need not worry about localized spaces here because the kernels of these operators are compactly supported. These results are not corollaries of the theorems on stratified groups; rather, one must repeat the proofs with appropriate modifications.) Presumably a similar theory of $\Gamma_{\alpha}$ spaces on $\tilde{U}$ can be developed, but this has not been carried out in 
general. See, however, [8] for the case of strongly pseudoconvex hypersurfaces.

These results give the sharp regularity properties of $\tilde{L}$, and it now remains to descend back to the original operator $L$ on the open set $U \subset M$ of Theorem 10. First we consider some function spaces on $U$. We still have the classical spaces $L_{\alpha}^{p}$ and $\Lambda_{\alpha}$ on $U$, and we define $S_{k}^{p}$ as before using the vector fields $X_{1}, \ldots, X_{n}$ on $U$. (Note that the character of $S_{k}^{p}$ may change from point to point. For instance, in our example (3), $S_{k}^{p}(\mathrm{loc})$ agrees with $L_{k}^{p}(\mathrm{loc})$ away from $x=0$, but it is strictly larger than $L_{k}^{p}(\mathrm{loc})$ along $x=0$.) We say that an operator on functions on $U$ is smoothing of order $\lambda$ if it maps $L_{\alpha}^{p}, \Lambda_{\alpha}$ and $S_{k}^{p}$ boundedly into $L_{\alpha+(\lambda / m)}^{p}, \Lambda_{\alpha+(\lambda / m)}$, and $S_{k+\lambda}^{p}$, respectively, for $\alpha>0,1<p$ $<\infty$, and $k=0,1,2, \ldots$

Recall that $\tilde{U}=U \times U^{\prime}$, where $U^{\prime}$ is a neighborhood of 0 in $\mathbf{R}^{k}$, and that $\pi: \tilde{U} \rightarrow U$ is the projection. We thus have a canonical lifting map $E$ from functions on $U$ to functions on $\tilde{U}$ defined by $E f=f \circ \pi$. We define a left inverse $R$ to $E$ as follows. If $\xi \in \tilde{U}$, write $\xi=(x, t)$ with $x \in U, t \in U^{\prime}$. Fix $\zeta \in C_{0}^{\infty}\left(U^{\prime}\right)$ with $\int \zeta(t) d t=1$, where $d t$ is Lebesgue measure on $\mathbf{R}^{k}$. Then if $f$ is a function on $\tilde{U}$, set

$$
R f(x)=\int f(x, t) \zeta(t) d t .
$$

Next, in the definition (5) of the kernel of the parametrix $\tilde{T}$ for $\tilde{L}$, take $\psi$ to be of the form $\psi(x, t)=\phi(x) \zeta^{\prime}(t)$, where $\phi \in C_{0}^{\infty}(U), \zeta^{\prime} \in C_{0}^{\infty}\left(U^{\prime}\right)$, and $\zeta^{\prime}=1$ on the support of $\zeta$. Then define the operators $T, S, S^{\prime}$ on functions on $U$ by

$$
T f=R \tilde{T} E f, \quad S f=L T f-\phi f, \quad S^{\prime} f=T L f-\phi f .
$$

THEOREM 12. $T$ is smoothing of order 2 , and $S$ and $S^{\prime}$ are smoothing of order 1 .

Thus $T$ is a parametrix for $L$; more precisely, $T$ inverts $L$ modulo a smoothing operator on any open set where $\phi=1$. Since $U$ was any sufficiently small open set in $M$, and $\phi \in C_{0}^{\infty}(U)$ was arbitrary, we can conclude the following regularity theorem.

THEOREM 13. Suppose $L=\sum_{1}^{n} X_{j}^{2}$ where $X_{1}, \ldots, X_{n}$ satisfy the Hörmander condition of order $m$, and $L u=f$. If $f$ is locally in $L_{\alpha}^{p}, \Lambda_{\alpha}$, or $S_{k}^{p}(\alpha>0,1<p$ $<\infty, k=0,1,2, \ldots)$, then $u$ is locally in $L_{\alpha+(2 / m)}^{p}, \Lambda_{\alpha+(2 / m)}$, or $S_{k+2}^{p}$, respectively.

As we mentioned above, these arguments can be generalized to handle operators of the form $\sum_{1}^{n} X_{j}^{2}+X_{0}$ where $X_{0}, X_{1}, \ldots, X_{n}$ satisfy a Hörmander condition, as well as the Laplacians $\square_{b}$ of the $\bar{\partial}_{b}$ complex. For the latter, one obtains analogues of the results of [8] on arbitrary hypersurfaces satisfying Kohn's condition $Y(q)$, with no restriction on the metric. These are of the same character as Theorem 13.

The lifting process described above was designed for maximum generality, particularly in its universal use of the free nilpotent group $G_{n, m}$. However, in some situations one can obtain more precise results more cheaply. We shall 
now briefly describe one such situation; for the details, see [6].

Let $V$ be a finite-dimensional real vector space with a family of dilations $\left\{\gamma_{r}\right\}$ defined by $\gamma_{r}=\exp (A \log r)$, where $A$ is a diagonalizable linear transformation of $V$ whose eigenvalues $\alpha_{j}$ are positive integers with $\min \left\{\alpha_{j}\right\}=1$ and $\max \left\{\alpha_{j}\right\}=m>1$. Let $X_{1}, \ldots, X_{n}$ be real vector fields with polynomial coefficients on $V$ which are homogeneous of degree 1 with respect to the dilations $\left\{\gamma_{r}\right\}$ and satisfy the Hörmander condition of order $m$. (Example: Take $V=\mathbf{R}^{2}, \gamma_{r}(x, y)=\left(r x, r^{2} y\right)$, and $X_{1}, X_{2}$ given by (3).) Then the $X_{j}^{\prime}$ 's generate a stratified Lie algebra $g$ of vector fields on $V$. Let $G$ be the corresponding group; if $X \in \mathfrak{g}$, we denote the corresponding left-invariant vector field on $G$ by $\tilde{X}$. As it turns out, there is a natural transitive right action of $G$ on $V$, and the isotropy subgroup of 0 is $H=\exp (\mathfrak{h})$, where $\mathfrak{h}=\left\{X \in \mathfrak{g}:\left.X\right|_{0}=0\right\} . V$ can thus be identified with the homogeneous space $H \backslash G$, and if $\pi: G \rightarrow H \backslash G$ $\cong V$ is the projection, we have $\tilde{X}(f \circ \pi)=(X f) \circ \pi$ for all $X \in g$ and $f \in C^{\infty}(V)$. The correspondence $X_{j} \rightarrow \tilde{X}_{j}$ is thus a lifting of the vector fields $X_{j}$ to left-invariant vector fields on $G$. Moreover, let $L=\sum_{1}^{n} X_{j}^{2}, \tilde{L}=\sum_{1}^{n} \tilde{X}_{j}^{2}$, and $\tilde{K}=$ the fundamental solution for $\tilde{L}$ given by Theorem 1 . Then the integral operator on $V$ whose kernel $K$ is defined by

$$
K(\pi(x), \pi(y))=\int_{H} \tilde{K}\left(y^{-1} h x\right) d h \quad(x, y \in G, d h=\text { Haar measure })
$$

inverts $L$ exactly.

The ideas we have discussed can also be applied to the $\bar{\partial}$ operator. In a forthcoming monograph [10]. Greiner and Stein apply nilpotent analysis to obtain a parametrix for the $\bar{\partial}$-Neumann problem for $(0,1)$-forms on a domain $D$ with strongly pseudoconvex boundary $M$, assuming that the metric is of the sort described in [8]. The idea is as follows. Recall that the problem is to solve

$$
\square \phi=\psi, \quad \nu \phi=\nu \bar{\partial} \phi=0,
$$

where $\psi$ is a given $(0,1)$-form on $D$. First one finds a form $\phi^{\prime}$ satisfying $\square \phi^{\prime}=\psi, \quad \nu \phi^{\prime}=0$, by solving a classical Dirichlet problem, which yields coercive estimates for $\phi^{\prime}$. Setting $\zeta=\phi-\phi^{\prime}$, then, one is reduced to solving $\square \zeta=0, \nu \zeta=0, \nu \bar{\partial} \zeta=\theta$, where $\theta=-\nu \bar{\partial} \phi^{\prime}$ is a given form on $M$. If one can discover what the restriction $\zeta_{0}$ of $\zeta$ to $M$ must be in order to satisfy these conditions, one can recover $\zeta$ from $\zeta_{0}$ by applying the Poisson integral for $\square$. But there is a first-order pseudodifferential operator $N$ on $M$ such that $\nu \bar{\partial} \zeta=N \zeta_{0}$ for solutions of $\square \zeta=0$. Moreover, Greiner and Stein construct another first-order pseudodifferential operator $T$ on $M$ such that

$$
T N=\square_{b}+\text { lower order terms. }
$$

Hence, to solve $\nu \bar{\partial} \zeta=\theta$ approximately, it suffices to solve $\square_{b} \zeta_{0}=T \theta$ approximately, and this is accomplished by applying the parametrix for $\square_{b}$ constructed in [8]. This process also automatically yields $\nu \zeta=0$.

The upshot of all this, then, is that one obtains an approximate solution to the problem (6), the explicit construction of which allows one to prove a 
variety of sharp estimates and regularity theorems for (6). These are much the same character as the results for $\square_{b}$ proved in [8] and [22].

In the same spirit, though by different methods, Krantz [18], [19] has obtained a number of sharp estimates for Henkin's solution of $\partial \phi=\psi$, where $\psi$ is a $(0,1)$-form on a bounded domain with strongly pseudoconvex boundary, in terms of $L^{p}$ spaces and nonisotropic Lipschitz spaces similar to our spaces $\Gamma_{\alpha}$.

One aspect of harmonic analysis on nilpotent groups which we have not mentioned at all is representation theory, although representations play a peripheral role in [8] and [22]. In general, representation theory seems to be a rather unwieldy tool for the kinds of problems we have been considering. However, Rockland [21] has recently proved an interesting hypoellipticiiy theorem in the context of representations of the Heisenberg group $H_{n}$ :

THEOREM (ROCKLAND). Let $L$ be a left-invariant differential operator on $H_{n}$ which is homogeneous with respect to the standard dilations on $H_{n}$. Then $L$ and its transpose are hypoelliptic if and only if $d \pi(L)$ has a bounded two-sided inverse for every nontrivial irreducible unitary representation $\pi$ of $H_{n}$, where $d \pi$ is the representation of the universal enveloping algebra of $H_{n}$ corresponding to $\pi$.

This theorem is the natural analogue for the $H_{n}$ of the fact that a homogeneous constant-coefficient differential operator on $\mathbf{R}^{n}$ is hypoelliptic if and only if it is elliptic.

ADDED IN PROOF. Recently Folland [26] and Krantz [27] have obtained some new results on the structure of Lipschitz classes on nilpotent groups, and Metivier [28] has used nilpotent analysis to study the asymptotic behavior of the spectra of operators of Hörmander type. For further background on the material discussed in the present paper, the reader may consult Goodman [29] and Stein [30].

\section{REFERENCES}

1. A. P. Calderón and A. Zygmund, Singular integral operators and differential equations, Amer. J. Math. 79 (1957), 901-921. MR 20 \#196.

2. R. R. Coifman and G. Weiss, Analyse harmonique non-commutative sur certains espaces homogènes, Lecture Notes in Math., vol. 242, Springer-Verlag, Berlin and New York, 1971.

3. G. B. Folland, A fundamental solution for a subelliptic operator, Bull. Amer. Math. Soc. 79 (1973), 373-376. MR 47 \#3816.

4. - Subelliptic estimates and function spaces on nilpotent Lie groups, Ark. Mat. 13 (1975), 161-207.

5. - Introduction to partial differential equations, Math. Notes, no. 17, Princeton Univ. Press, Princeton, N.J., 1976.

6. _ On the Rothschild-Stein lifting theorem, Comm. Partial Differential Equations 2 (1977), 165-191.

7. G. B. Folland and J. J. Kohn, The Neumann problem for the Cauchy-Riemann complex, Ann. of Math. Studies, no. 75, Princeton Univ. Press, Princeton, N.J., 1972.

8. G. B. Folland and E. M. Stein, Estimates for the $\bar{\partial}_{b}$ complex and analysis on the Heisenberg group, Comm. Pure Appl. Math. 27 (1974), 429-522. MR 51 \# 3719.

9. R. Goodman, Lifting vector fields to nilpotent Lie groups (to appear).

10. P. C. Greiner and E. M. Stein, Estimates for the $\bar{\partial}$-Neumann problem, Princeton Univ. Press, Princeton, N.J., 1977. 
11. L. Hörmander, Hypoelliptic second order differential equations, Acta Math. 119 (1967), 147-171. MR 36 \#5526.

12. A. W. Knapp and E. M. Stein, Intertwining operators for semi-simple groups, Ann. of Math. (2) 93 (1971), 489-578.

13. J. J. Kohn, Harmonic integrals on strongly pseudo-convex manifolds. I, II, Ann. of Math. (2) 78 (1963), 112-148; ibid. (2) 79 (1964), 450-472. MR 27 \#2999, 34 \#8010.

14. - Boundaries of complex manifolds, Proc. Conf. on Complex Analysis (Minneapolis, 1964), Springer-Verlag, Berlin and New York, 1965, pp. 81-94. MR 30 \# 5334.

15. - Pseudo-differential operators and hypoellipticity, Proc. Sympos. Pure Math., vol. 23, Amer. Math. Soc., Providence, R.I., 1973, pp. 61-69. MR 49 \#3356.

16. J. J. Kohn and L. Nirenberg, Non-coercive boundary value problems, Comm. Pure Appl. Math. 18 (1965), 443-492. MR 31 \#6041.

17. A. Korányi and S. Vági, Singular integrals on homogeneous spaces and some problems of classical analysis, Ann. Scuola Norm. Sup. Pisa 25 (1971), 575-648.

18. S. G. Krantz, Optimal Lipschitz and $L^{p}$ regularity for the equation $\bar{\partial} u=$ fon strongly pseudoconvex domains, Math. Ann. 219 (1976), 233-260.

19. - Structure and interpolation theorems for certain Lipschitz spaces and estimates for the J equation, Duke Math. J. 43 (1976), 417-439.

20. I. I. Pjateckiî-Šapiro, Geometry of classical domains and theory of automorphic functions, Gosudarstv. Izdat. Fiz.-Mat. Lit., Moscow, 1961. (Russian) MR 25 \#231.

21. C. Rockland, Hypoellipticity on the Heisenberg group: representation-theoretic criteria (to appear).

22. L. P. Rothschild and E. M. Stein, Hypoelliptic differential operators and nilpotent groups, Acta Math. 137 (1976), 247-320.

23. E. M. Stein, Singular integrals and differentiability properties of functions, Princeton Univ. Press, Princeton, N.J., 1970. MR 44 \# 7280.

24. - Some problems in harmonic analysis suggested by symmetric spaces and semi-simple groups, Proc. Internat. Congress Math. (Nice, 1970), vol. 1, Gauthier-Villars, Paris, 1971, pp. 173-189.

25. - Singular integrals and estimates for the Cauchy-Riemann equations, Bull. Amer. Math. Soc. 79 (1973), 440-445. MR 47 \#3851.

26. G. B. Folland, Lipschitz classes and Poisson integrals on stratified groups (to appear).

27. S. G. Krantz, Characterizations of certain Lipschitz classes and subelliptic estimates for differential operators on nilpotent groups (to appear).

28. G. Metivier, Fonction spectrale et valeurs propres d'une classe d'opérateurs non elliptiques, Comm. Partial Differential Equations 1 (1976), 467-519.

29. R. Goodman, Nilpotent Lie groups: structure and applications to analysis, Lecture Notes in Math., vol. 562, Springer-Verlag, Berlin and New York, 1976.

30. E. M. Stein; Singular integral operators and nilpotent groups, Differential Operators on Manifolds (C.I.M.E., Varenna, 1975), Edizioni Cremonese, Rome, 1975, pp. 148-206.

Department of Mathematics, University of Washington, Seattle, Washington 98195 